\title{
Resume Parser with Natural Language Processing
}

This paper was downloaded from TechRxiv (https://www.techrxiv.org).

\section{LICENSE}

CC BY 4.0

SUBMISSION DATE / POSTED DATE

$25-12-2021$ / 29-12-2021

\section{CITATION}

Sroison, Pornphat; Chan, Jonathan H. (2021): Resume Parser with Natural Language Processing. TechRxiv. Preprint. https://doi.org/10.36227/techrxiv.17641604.v1

$\mathrm{DOI}$ 


\section{Resume Parser with Natural Language Processing}

\author{
Pornphat Sroison \\ School of Information Technology \\ King Mongkut's University of Technology Thonburi \\ Bangkok, Thailand \\ pornphat.phat@mail.kmutt.ac.th
}

\author{
Assoc.Prof.Dr. Jonathan Hoyin Chan \\ School of Information Technology \\ King Mongkut's University of Technology Thonburi \\ Bangkok, Thailand \\ jonathan@sit.kmutt.ac.th
}

\begin{abstract}
Because of the advancement of the online recruiting system. On the job application website, candidates can easily upload their resume. Resulting in a huge number of resumes being submitted. As a result, the human resource department faces a challenge in recruiting new employees and reviewing a large number of resumes. Furthermore, candidates who upload their resumes come in a variety of formats, including writing style, fonts, font sizes, colors, and etc. Human resource departments face a challenging problem in reading the entire resume that candidates upload and selecting the best candidate for the job position. So, for this project, I propose to resume parser by using natural language processing to assist the human resource department or recruiter in extracting the detailed information of the resume that is needed to proceed with the applicant's process and also reduce errors in the work. This proposed system consists of three steps to parsing resume: 1) Receive resume files from candidate 2) Convert resume file to the text format 3) Extracting necessary information. The system will extract only relevant data that is necessary for the selection of the resume: name, consisting of first name and last name, position applied for, university, degree, skill, work experience, email and phone number. In addition, the system can also display the result percentage of similarity between resumes and job descriptions. To make it easier for recruiters to make recruitment selection
\end{abstract}

Keywords-Resume Parser, Extracting Information, Matching, Human Resource, Employer, Natural Language Processing

\section{INTRODUCTION}

Nowadays, large companies and corporations have a large number of candidates that are applying for jobs via recruitment websites. Companies' human resource departments or recruiters are responsible for screening through a large number of resumes every day. This is not a job for humans because screening numerous resumes and selecting applicants for an interview takes a lot of time and can result in errors due to human fatigue. Resumes are unstructured data that differ from format of writing an email, web pages content and others data with defined structure. Applicants' resumes generally include a variety of information, as well as colors, fonts, presenting order, and literary styles in which they are written are different. Resumes are also available in various file formats, including '.txt,'.pdf,'.doc,'.docx,'.odt,'.rtf,' and etc. Those file types are usually used by job candidates. As a result, an automated intelligent system based on natural language processing is required to extract all of the information from unstructured resumes and a variety of data sources. The method for parse resumes is converting all resumes to a similar structured format and selecting only the information that is relevant to screening, such as name, position, education, years of experience, work experience, certificates, email, phone number, and etc. Following that, parsed resume data with structured format will be saved in a database for future use.

\section{OBJECTIVE}

1) To use this technology that is based on natural language processing to assist the human resource department in screening resumes before conducting interviews.

2) To parsing and matching the similarities between a candidate's resume and job description makes the hiring process easier and more efficient.

3) To help reduce human error and fatigue in screening resumes.

\section{SCOPE}

Degree, field of study, and work experiences of candidates are essential types of information for recruiting by the human resource department. They also want this system to be able to rank or compare resumes to job descriptions provided from them to evaluate if there are any similarities. This will make it easy for them to work and make recruiting selections. As a result, where we have to deal with a lot of data, converting a resume into formatted text or structured information to make it easier to review, analyze, extract relevant data, and understand is an essential Keep your text and graphic files separate until after the text has been formatted and styled. Do not use hard tabs, and limit use of hard returns to only one return at the end of a paragraph. Do not add any kind of pagination anywhere in the paper. Do not number text heads-the template will do that for you.

Parse resume and match resume to job description are the two functions of this system. The first function is to parse resumes. The user must upload a resume of the candidate file in PDF or DOC format. This project supports only PDF and DOC format because they are the most popular for creating resumes nowadays. The system will read all text of the resume and extract only relevant data that is necessary for the selection of the resume: name (first name and last name), position applied for, university, degree, work experience, skill, email address and phone number. The second function is matching resumes to job descriptions to evaluate how similar they are. The user can upload a job description file and see the displayed result as a percentage of similarity between resume of candidate and job description. This system can reduce the HR department's time reading all text of a resume and reduce errors in the work.

\section{LITERAL REVIEW}

1) Resume Analyzer Using Text Processing

This literal review presents an effective Company Recommender System that uses text mining and machine learning algorithms to help recruiters select the best candidate for a specific job. When candidates upload their resumes, they are ranked according to the company's 
requirements. The ranking can be used by the organization to select the best candidates.

This article's methodology and model will be provided in four steps: collecting resumes and searching for keywords stored in the information base on the resume text. Then, ranking and Categorization of candidates based on a rating score. Furthermore, this system may extract new keywords from resumes to expand the knowledge base further.

2) Automated extraction of information from Polish resume documents in the IT recruitment process

This literal review analyzes and discusses automated information retrieval for the IT industry's recruitment process. In terms of low resource language dictionaries and complicated linguistic relationships in Polish, the proposed approach implements a multi-module system.

This project uses the name of entity recognition, which is the most useful method for analyzing CVs. It's a semi-semantic analysis of the evaluated text that only recognizes specific words. It's an essential phase in getting the text's information content ready for processing.

\section{DATASET}

The data sets used in this project are divided into two datasets. First is a dataset of 200 resumes from GitHub consisting of names (first name and last name) and positions to apply for. Second is other datasets include global university and skills.

Table I. Number of datasets for each entity.

\begin{tabular}{|c|c|}
\hline Entity & Number of data \\
\hline Name & 205 \\
\hline Designation & 473 \\
\hline University & 829 \\
\hline Skills & 1,249 \\
\hline
\end{tabular}

The train dataset for parsing consists of 2 parts. The first part is content that specifies information about all text of resume in the text format. The second part is an entity annotation that will be in the form:

"annotation": \{"label":["text"],"points":[\{"start":

The number at the beginning of the word, "end": The number at the ending of the word, "text": "text of content"\}]\}. The example of the train dataset for parsing can be found on Fig.1

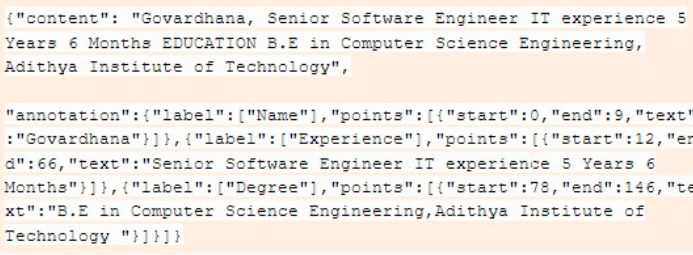

Fig 1: Example of the train dataset for parsing
1) Label is a label name that describes the type of word

2) Point Start and End is the number at the beginning and ending position of desired word from all text in the resume after converting the PDF or DOC file into text format.

3) Text is words in the content that is labeled

The entity name is a name about labeling or tagging that we will classify the desired word that we have specified position previously. In this project will use specify the 2 entities' names: name and designation

\section{Methodogy}

This project implements Named Entity Recognition, a part of Natural Language Processing that analyzes large amounts of unstructured human languages. The initial step in extracting information and topic modeling is NER extraction. The system reads the whole paragraph and highlights the text's key essential entity elements. Due to the resume text being an unstructured text into predefined categories, you can utilize Stanford NER or Spacy for this project.

Regular expressions have been used in this project, as well as regular expressions in scripts. A regular expression is a string of special characters that describes a search pattern by matching a character pattern to the string being searched. Regular expressions consist of literal symbols and special character combinations known as tokens, which indicate non-printable characters, symbols of a specific type, and the instructions for the regular expression engine. It is a formal language theory and theoretical computer science technique.

\section{A. PDF and DOC to text conversion}

This project uses PyMuPDF library to convert PDF files to text format and python-docx library to convert Doc, Docx file to text format.

\section{B. Named Entity Recognition (NER)}

Extracting name (first name and last name) and designation. This project uses the PKL or Pickle format for the train dataset. Pickle is a Python module that serializes objects so that they can be saved to a file and reloaded when the program calls them. Then, uses Named Entity Recognition (NER) for training model because this project is a finding and classifying text of resume that is an unstructured text into predefined categories by tagging dataset.

\section{Regular Expression}

Extracting the name of university by using regular expressions to search for keywords of university names such as University, School, College, Institute, etc. After that, searching for all the characters around those keywords.

Extracting degree or educational background by using regular expressions to search for keywords of university names such as Bachelor of, Master of, Doctor of, Degree, etc. After that, searching for all the characters around those keywords.

Extracting skill. First step is cleaning data by removing stop words that are a group of words that are regularly used in a language but contain relatively little valuable information, including punctuation on all text of 
resume. Then, search for each token in the skills database (.csv file). The final step is to create a bigram and trigram from a string of tokens or a skill database, which are often letters, syllables, or words, to identify a sequence of two or three nearby parts.

Extracting experience. First step is cleaning data by removing stop word and data preprocessing by word tokenization. Then, parse regular expressions by using chunk sequences of proper nouns $(\{<\mathrm{NNP}>+\})$. The final step is to search the word 'experience' in the chunk and then print out the text after 'experience' word in the line.

D. Regular Expressions in Scripts (Regex Scripts)

Extracting phone number by using Regex Scripts to extract phone number: ' $\backslash \backslash \backslash \backslash(]$ ? [1-9] [0-9. $\backslash-\backslash(\backslash)]\{8\},[0-$ 9]'. It works with standard phone numbers, including country and area codes for most international numbers.

Extracting email by using Regex Scripts to Extract emails address: '[a-z0-9\.।-+ ]+@ [a-z0-9\.।-+ ]+\. [a-z]+'. It Works with all standard email addresses as long as the email uses standard English and @ characters.

For the purpose of matching a resume to a job description that can be compared to see how similar they are. The percentage of similarity will be displayed as the result. The way of comparison is importing a library from scikit-learn (feature extraction) that can construct a count vector object to get a count of each word in the text and importing count vectorization. Then, using cosine similarity, determine how similar two documents are.

\section{RESULT}

The proposed system's results are shown in this part, which include extracting name, designation, university, degree, skills, experience, email and phone number using Named Entity Recognition to develop a model and Regular Expression to extract the data. Another feature of this system is that it compares the Resumes and job description of the applicant. The similarity of the outcomes is expressed as a percentage. Fig. 2, 3, and 4 show the entire system's results.

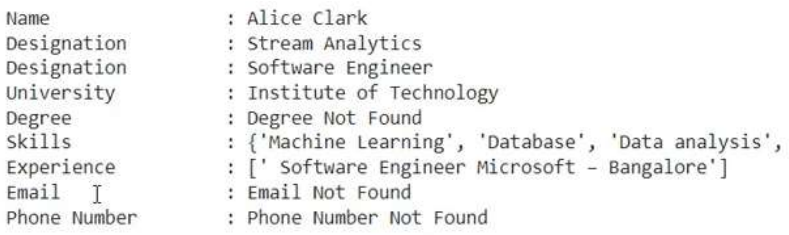

Fig 2: The example results of parser resume that consist of name, designation, university, degree, skills, experience, email and phone number

[23] \#Function that's going to tell how similar this text is from sklearn.metrics.pairwise import cosine_similarity print("'nsimilarity score:") print(cosine_similarity(count_matrix))

$$
\begin{aligned}
& \text { Similarity Score: } \\
& \text { [ [ } 1.0 .58789118] \\
& {\left[\begin{array}{lll}
0.58789118 & 1 .
\end{array}\right]}
\end{aligned}
$$

Fig 3: The example results of cosine similarity score that compares between resume of candidate and job description. \#get the match perpentage

matchPercentage $=$ cosine_similarity $($ count_matrix $)[\theta][1]^{*} 100$

matchpercentage $=$ round $($ matchPercentage, 2$)$ \#round to two decimal places print("Your resume matches about " + str(matchpercentage)+ "\% of the job description")

Your resume matches about $58.79 \%$ of the job description

Fig 4: The example results of cosine similarity score with percentage that compares between resume of candidate and job description.

\section{VIII.LIMITATION}

Because of the data extraction limitations, it includes some data that cannot be processed, such as the year of graduation and date of birth, which makes it difficult to determine which class it is because the resume mentioned many dates or years. In addition, there is not enough dataset in this project, and the information extracted does not cover all the details of the resume, such as experience. It can only retrieve a little amount of data that is closely connected to the word "experience." As a result, data retrieval problems are possible.

Resume parsing is also sensitive to ethical restrictions. Because of this system, the result will be a text input only. As a result, this approach is only suitable for screening some positions. For example, a graphic designer position or other design positions that require a visual preview of the work, an image as evidence of work, and consideration of the resume's beauty and color may not be appropriate for this system. This system's bias appears to be causing firms to lose employees.

\section{CONCLUSION}

Because the online recruiting system has progressed, a large number of resumes were submitted. Consequently, hiring new employees and reviewing a large number of resumes is a challenge for the human resource department or employer. Therefore, this system has helped employers by using an automated intelligent system based on natural language processing. This system can convert various formats of resumes to text format and can extract some important information successfully. It is also possible to compare the applicant's resume and the job description to see the percentage of similarity as well. This system can assist the human resource department or employer in screening resumes before conducting interviews and finding the best candidate for the job position.

\section{FurTher DEVELOPMENT}

This project intends to provide more datasets for training in the future because the existing datasets are insufficient for applications such as designation, university, skill, etc. For future website development. This project will apply the model to the website and add a function to view the applicant's resume file or portfolio if the employer or human resource department are interested. To support the selection of resumes in all positions. After the user confirms this candidate, the resume is saved in a NoSQL database to be used as a future dataset, with the resumes being ranked based on the percentage of similarity between the applicant's resume and the job description. 
To assist candidates, they can upload their resumes to an online recruitment website to double-check the information and compare the percentage of similarities between their resumes and the job description to help them decide whether to apply for a position.

\section{REFERENCES}

[1] What is resume parsing: Retrieved from, https://www.smartrecruiters.com/resources/glossary/resumeparsing/

[2] NLP Based Resume Parser using BERT in Python: Retrieved from, https:/www.pragnakalp.com/case-study/nlp-resume-parser-bertpython/

[3] NLP based resume parser in Python (Beta): Retrieved from, https://demos.pragnakalp.com/resume-parser/

[4] World University Ranking 2016: Retrieved from, https:/data.world/hhaveliw/world-university-ranking2016? fbclid=IwAR01WBDbntwc7K3NRkHpc1XCp8WcESQEV MR2zXCXD8R31f-NTwJv1DZ7mWY

[5] Resume Parser: Retrieved from, https://github.com/OmkarPathak/ResumeParser

[6] Resume and CV Summarization and Parsing with Spacy in Python: Retrieved from, https:/github.com/laxmimerit/Resume-and-CVSummarization-and-Parsing-with-Spacy-in-Python

[7] Automated-Resume-Screening-System Dataset: Retrieved from, https://github.com/JAIJANYANI/Automated-ResumeScreening-System

[8] How to extract email address, phone number and links from text: Retrieved from, https://zapier.com/blog/extract-links-email-phoneregex/

[9] Literature Reviews - Resume Analyzer Using Text Processing: Retrieved from, https://jespublication.com/upload/2020-110557.pdf

[10] Literature Reviews - Automated extraction of information from Polish resume documents in the IT recruitment process: Retrieved from, https://www.sciencedirect.com/science/article/pii/S18770509 2101749X 